selective serotonin reuptake inhibitors (SSRIs) are the most frequently cited environmental risk factors.

We sought phenotypic and genetic associations with microscopic colitis in European patients enrolled in the UK Biobank.

Methods We undertook a genome-wide association study of 483 cases of microscopic colitis defined by ICD code K52.8 (other specified noninfective gastroenteritis and colitis) and 450616 controls. We tested 9527357 single nucleotide polymorphisms (SNPs) imputed using the haplotype reference consortium (HRC) reference panel. Association tests were performed using a linear mixed model (BOLT-LMM) including age, gender, study centre and chip as covariates. We also tested for associations with classical HLA alleles that were imputed using HLA*IMP 02.

Results Participants with microscopic colitis were older (61.9 [56.2-65.4] vs. controls 58.6 [50.5-63.8], p=5e-15); more frequently female $(65.6 \%$ [317/483] vs $54.2 \%$ [244531/450616], $\mathrm{p}=5 \mathrm{e}-07)$; more likely to smoke $(14.7 \%$ [71/483] vs $10.4 \%$ [46792/450616], $\mathrm{p}=0.003)$ and were more often also diagnosed with coeliac disease $(3.3 \%$ [16/483] and 0.4\% [1991/ 450616], $\mathrm{p}=7 \mathrm{e}-10$ ) than controls. In terms of drug factors, participants with microscopic colitis were more likely to have been exposed to proton-pump inhibitors (20.3\% [98/483] cases vs $10.3 \%$ [46397/450156] controls, $\mathrm{p}=9 \mathrm{e}-11$ ) than controls, but not aspirin/NSAIDs or SSRIs.

We found a genome-wide significant association signal within the HLA region. The lead SNP was rs2596560 (OR 0.64, 95\% CI: 0.57, 0.71, p=4e-9). Subsequent HLA imputation demonstrated that the signal was in linkage disequilibrium with the class I and II alleles that comprise the ancestral MHC 8.1 haplotype that has been linked with coeliac disease. Multivariable linear analyses showed that microscopic colitis $(\mathrm{p}=9 \mathrm{e}-08)$ and coeliac disease were independently associated with the rs2596560 SNP. There were no specific genetic associations seen in the subset of participants with microscopic colitis taking aspirin/NSAIDS, PPIs, or SSRIs.

Conclusions We have confirmed a genome-wide significant association for microscopic colitis in the HLA region. Further studies are needed to understand the role of this locus in the pathogenesis of microscopic colitis and larger drug exposed cohorts will need to be identified to explore possible pharmacogenetic associations.

\section{OTU-023 RANDOMISED TRIAL OF EPA AND ASPIRIN FOR COLORECTAL CANCER CHEMOPREVENTION: THE SEAFOOD POLYP PREVENTION TRIAL}

\footnotetext{
${ }^{1} \mathrm{M}$ Hull ${ }^{*},{ }^{2} \mathrm{~K}$ Sprange, ${ }^{2} \mathrm{~T}$ Hepburn, ${ }^{2} \mathrm{~W}$ Tan, ${ }^{2} \mathrm{~A}$ Shafyat, ${ }^{3} \mathrm{G}$ Clifford, ${ }^{3} \mathrm{C}$ Rees, ${ }^{2} \mathrm{R}$ Logan, ${ }^{4} \mathrm{P}$ Loadman, ${ }^{5} \mathrm{E}$ Williams, ${ }^{2} \mathrm{D}$ Whitham, ${ }^{2} \mathrm{~A}$ Montgomery, sea food Collaborative Group. ${ }^{1}$ University of Leeds; ${ }^{2}$ Nottingham; ${ }^{3}$ South Tyneside NHS Foundation Trust; ${ }^{4}$ Bradford; ${ }^{5}$ Sheffield
}

\subsection{6/gutjnl-2018-BSGAbstracts.363}

The omega-3 fatty acid eicosapentaenoic acid (EPA) and aspirin are candidate colorectal cancer (CRC) chemoprevention agents, which both have proof-of-concept for anti-CRC activity in man, aligned with an excellent safety profile.

Methods A randomised, placebo-controlled $2 \times 2$ factorial trial of EPA free fatty acid (FFA) $2 \mathrm{~g}$ daily (E; either as the
FFA or triglyceride [TG]) and/or aspirin $300 \mathrm{mg}$ daily (A) in 'high risk' patients $(\geq 3$ adenomas if one $\geq 10 \mathrm{~mm}$, or $\geq 5$ small adenomas) identified at screening colonoscopy in the English Bowel Cancer Screening Programme (BCSP). The primary endpoint was the adenoma detection rate (ADRa; the $\%$ with any adenoma) at one year surveillance colonoscopy. Secondary endpoints included mean number of adenomas per patient (MAP), 'advanced' ADRa, adenoma location (right/left) and type (conventional/serrated). Analysis was on an intention-totreat basis using an 'at the margins' approach, adjusted for BCSP site and repeat endoscopy at baseline.

We recruited 709 participants (80\% male, mean[SD] 65[5] years, $82 \%$ BMI $>25 \mathrm{Kg} / \mathrm{m}^{2}$ ). The four treatment groups (E $+\mathrm{A} n=177$; $\mathrm{E} n=178$; A $n=176$; placebo $\mathrm{n}=176$ ) were wellmatched at baseline. There were no differences in EPA levels or tolerability between FFA and TG users. Overall, ADRa was $62 \%$, with no evidence of any effect for EPA (risk ratio 0.98 [95\% CI 0.87-1.12]) or aspirin (0.99 [0.87-1.12]). Aspirin use was associated with reduced total MAP (incidence rate ratio 0.78 [95\% CI $0.68-0.90]$ ), with evidence of an effect on serrated (0.46 [0.25-0.87]) and right-sided (0.73 [0.61-0.88]) lesions. Evidence that EPA reduced MAP was restricted to conventional (0.86 [0.74-0.99]), left-sided (0.75 [0.60-0.94]) adenomas, but not total MAP (0.91 [0.79-1.05]). EPA and aspirin treatment were well tolerated with an excess of mildmoderate GI adverse events (AEs), especially in the $\mathrm{E}$ arm. There were 6 bleeding AEs across the treatment arms.

Neither EPA nor aspirin treatment was associated with reduction in the ADRa in 'high risk' patients. Secondary analyses revealed no evidence that EPA was effective in reducing the total number of adenomas, but there was some evidence for efficacy of aspirin. Both agents displayed effects on MAP, which were adenoma type- and site-specific, compatible with known anti-(proximal) CRC activity of aspirin. Best use of EPA and aspirin may need a precision medicine approach to adenoma recurrence. ISRCTN05926847 - This project was funded by the EME Programme, an MRC and NIHR partnership. The views expressed in this publication are those of the author(s) and not necessarily those of the MRC, NHS, NIHR or the DoH.

\section{OTU-024 IMPACT OF GFOBT SCREENING IN ENGLAND ON COLORECTAL CANCER MORTALITY} ${ }^{1,2}$ Joanne R Morling*, ${ }^{1}$ Caroline J Chapman, ${ }^{1,2}$ Richard F Logan. ${ }^{1}$ Bowel Cancer Screening
Programme (Eastern Hub), Nottingham, UK; ${ }^{2}$ Division of Epidemiology and Public Health,
University of Nottingham, Nottingham, UK

\subsection{6/gutjnl-2018-BSGAbstracts.364}

Introduction Colorectal cancer (CRC) screening using biennial gFOBT was introduced in England in September $2006^{1}$ and by 2010 was being offered $>90 \%$ of $60-69$ year olds, rising to $>95 \%$ of $60-74$ year olds by 2014 . Uptake of screening has shown no substantial change since 2006 and in 2016 was $58 \%$ for the whole of England. This study seeks to examine the trends in CRC mortality and ascertain any impact of screening.

Methods Data for the period 2001-2016 was extracted from the ONS website (www.ons.gov.uk) and CRC mortality rates by 5 year age bands from age 45 calculated. CRC was defined 